\title{
The Analysis of the Contribution of Human Factors to the In-flight Loss of Control Accidents
}

\author{
Ersin Ancel ${ }^{*}$ \\ National Institute of Aerospace, Hampton, VA, 23666 \\ Ann T. Shih \\ NASA Langley Research Center, Hampton, VA, 23681
}

\begin{abstract}
In-flight loss of control (LOC) is currently the leading cause of fatal accidents based on various commercial aircraft accident statistics. As the Next Generation Air Transportation System (NextGen) emerges, new contributing factors leading to LOC are anticipated. The NASA Aviation Safety Program (AvSP), along with other aviation agencies and communities are actively developing safety products to mitigate the LOC risk. This paper discusses the approach used to construct a generic integrated LOC accident framework (LOCAF) model based on a detailed review of LOC accidents over the past two decades. The LOCAF model is comprised of causal factors from the domain of human factors, aircraft system component failures, and atmospheric environment. The multiple interdependent causal factors are expressed in an Object-Oriented Bayesian belief network. In addition to predicting the likelihood of LOC accident occurrence, the system-level integrated LOCAF model is able to evaluate the impact of new safety technology products developed in AvSP. This provides valuable information to decision makers in strategizing NASA's aviation safety technology portfolio. The focus of this paper is on the analysis of human causal factors in the model, including the contributions from flight crew and maintenance workers. The Human Factors Analysis and Classification System (HFACS) taxonomy was used to develop human related causal factors. The preliminary results from the baseline LOCAF model are also presented.
\end{abstract}

\section{Introduction}

A LTHOUGH the overall accident rate has decreased, in-flight loss of control (LOC) is the leading cause of fatal accidents. The Boeing statistical summary of commercial jet aircraft accidents ${ }^{1}$ shows that 1841 lives (about $37 \%$ of total fatalities) were lost in 20 (out of 87) fatal LOC accidents worldwide in the years 2001-2010. Similarly, an examination of the National Transportation Safety Board (NTSB) accident database ${ }^{\ddagger}$ reveals that LOC accidents represent more than $10 \%$ of all US-based commercial airlines accidents and more than $50 \%$ of all fatalities over the past two decades. Furthermore, findings in the LOC accident reports suggest the source of causality is centered around human errors.

In addition to the current contributing factors leading to LOC, the Next Generation Air Transportation System (NextGen) as envisioned in the 2025 timeframe is expected to bring forth potential future causes, contributing to LOC with decreased separation distance and increased National Airspace System (NAS) capacity. The NASA Aviation Safety Program (AvSP) conducted a high-level qualitative analysis of future safety risks ${ }^{2}$, identifying LOC as one of the most critical future risks. A better understanding of LOC causes and prevention strategies are necessary to help reduce the fatality rate and to keep the accident rate low in the foreseeable NAS. To this end, the AvSP Aviation Safety Analysis Team (ASAT) has been asked to develop a system-level integrated LOC model, not only to predict the likelihood of future LOC, but, more importantly, to evaluate the impact of new safety technology product insertions on LOC.

This paper discusses the approach used to develop a generic integrated LOC accident framework (LOCAF) model from the causal factor analysis of LOC accidents for the time period 1987-2009. This integrated LOC model

\footnotetext{
* Research Engineer, E-mail: ersin.ancel@ nianet.org

$\dagger$ Aerospace Engineer, Vehicle Analysis Branch, E-mail: ann.t.shih@nasa.gov

${ }^{*}$ http://www.ntsb.gov/aviationquery/index.aspx
} 
was constructed as an Influence Diagram (ID), an extension of Bayesian belief network (BBN), using Hugin ${ }^{\S}$, a commercially available software package. The causal factor analysis focuses on human related contributing factors including pilots and maintenance workers. The Human Factors Analysis and Classification System (HFACS) taxonomy was used for human related causal factors in the BBN-based LOC model. The decision nodes in the model were used to represent proposed new safety technology products currently being developed in the AvSP Vehicle Systems Safety Technologies (VSST) project, System-Wide Safety and Assurance Technologies (SSAT) project, and Atmospheric Environment Safety Technologies (AEST) project.

\section{LOC Accidents Database Review}

In order to investigate current and future LOC accidents, a set of historical data was considered for review and categorization. The Aviation Safety Analysis Team (ASAT) ${ }^{2}$ at NASA periodically obtains a copy of the NTSB accident/incident database from Aviation Safety Information Analysis and Sharing (ASIAS) ${ }^{* *}$. All accidents between 1987 and 2008 involving fixed-wing commercially built aircraft operating under Federal Aviation Regulations (FAR) Part 121, Part 135 or Part 91 have been assigned occurrence categories based on the taxonomy developed by the Commercial Aviation Safety Team/International Civil Aviation Organization (CAST/ICAO) Common Taxonomy Team (CICTT) ${ }^{3,4}$. Out of this database, the LOC-I accidents for FAR Part 121 and Part 135 were selected. Additional accidents for 2009 were acquired from the NTSB database and categorized using the CICTT taxonomy.

A total of 316 accidents from Part 121 and Part 135 for the years 1987-2009 constitute the initial LOC accident database for the current study. The initial list of LOC accidents was refined based on a set of analysis ground rules and assumptions, such as restricting consideration to accidents occurring in the U.S with maximum take-off weights (MTOW) exceeding 12,500 lbs. As a result, the final accident list contains 54 LOC accident cases with 1429 fatalities, 154 serious injuries, and 283 minor injuries for 1987-2009 timeline, which covers $47.7 \%$ fatalities of all Part 121 and 135 accidents within the same timeframe.

In order to categorize the 54 LOC cases cited above, the authors mainly employed NTSB accident reports. However, for the cases where the sole information source is the less detailed NTSB factual report, an additional source of information was found in the Aviation Safety Analysis and Functionality Evaluation (ASAFE) program. The ASAFE program uses a modified version of the Human Factors Analysis and Classification System (HFACS) ${ }^{5}$ taxonomy and its maintenance extension (HFACS-ME) ${ }^{6}$, assigning corresponding causal factor codes via the designated computer program. The additional information in ASAFE allowed the authors to further classify the LOC accidents associated with human errors within the provided timeframe.

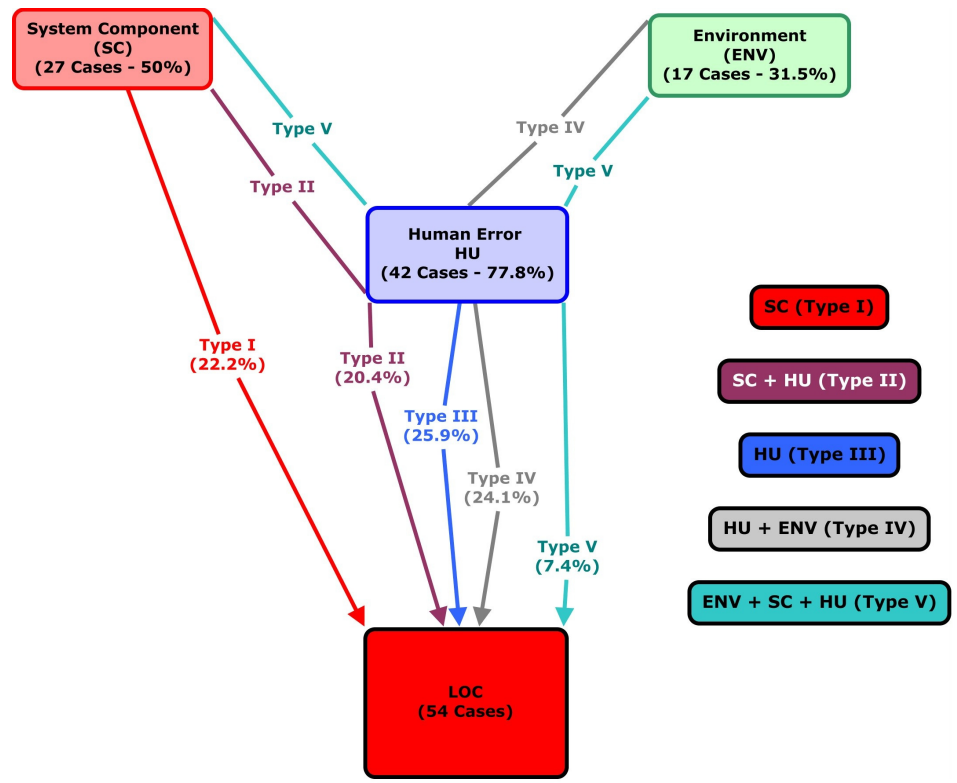

Figure 1. LOC Primary accident cause distribution.

\section{Generalized LOC Accident Types}

As previously mentioned, the database review allowed authors to construct a generalized LOC Accident Framework (LOCAF). Given the information provided from the CICTT categorization, NTSB accident and factual reports, and ASAFE, these 54 LOC accident cases were reviewed for the causal and contributing accident factors from three main perspectives; namely, the aircraft system, human, and external environment. The aircraft system components include System Component (SC) related accidents arising from maintenance, design and vehicle system issues. The HUman (HU) component includes flight crew and ground personnel. The external ENVironment (ENV) relates to atmospheric disturbances, such as icing, turbulence, etc. All the accidents contain

$\S$ www.hugin.com - Hugin Expert A/S

*** http://www.asias.faa.gov/portal/page/portal/asias_pages/asias_home/ 
either a single or a combination of these primary components, and were grouped into five main accident categories.

The number of occurrences of these five main accident categories is given in Fig. 1. Out of the 54 accidents, only 12 accidents are directly related to an aircraft system or component issue (Type I - SC, 22.2\%). The remaining 42 accidents (78.2\%) include human error, either directly or indirectly. In 11 of the cases, both the flight crew and the aircraft systems were found to be contributors to the accident (Type II - SC+HU, 20.4\%). The human error (both flight crew or ground personnel induced) was observed in 14 accidents (Type III - HU, 25.9\%). Out of the human related accidents, $3(5.5 \%)$ were related to ground personnel, and $11(20.4 \%)$ were associated with flight crew deficiencies. Out of all cases, 17 accidents were related to a combination of weather related disturbances, coupled with flight crew errors (Type IV - HU+ENV, 31.5\%). Finally, four accidents involved all three components, the human, environment, and system component issues (Type V - HU+ENV+SCF, 7.4\%).

Although not shown, inappropriate Air Traffic Management (ATM) guidance was associated with 5 accidents, cited as findings by the NTSB reports, but not determined as primary factor or cause. Four of the ATM related accidents were encountered in environment category (ground/inflight icing, wake turbulence, and conducive weather related) and in one accident, ATM guidance contributed to flight crew's task fixation.

The next step in the research was to develop a generalized LOC accident framework (LOCAF) embarking from the classification given above, which is able to demonstrate the individual accident cases covered in the dataset while identifying the interaction among the three major components, human, aircraft systems, and the environment.

\section{Generalized LOC Accident Framework (LOCAF)}

The generalized LOCAF was mainly based on an extensive review of the historical accidents given in the database review section. LOCAF provides the foundation for the LOC model. Fig. 2 illustratres the accident framework as a generalized interaction of various factors given in the boxes, leading to a LOC accident.

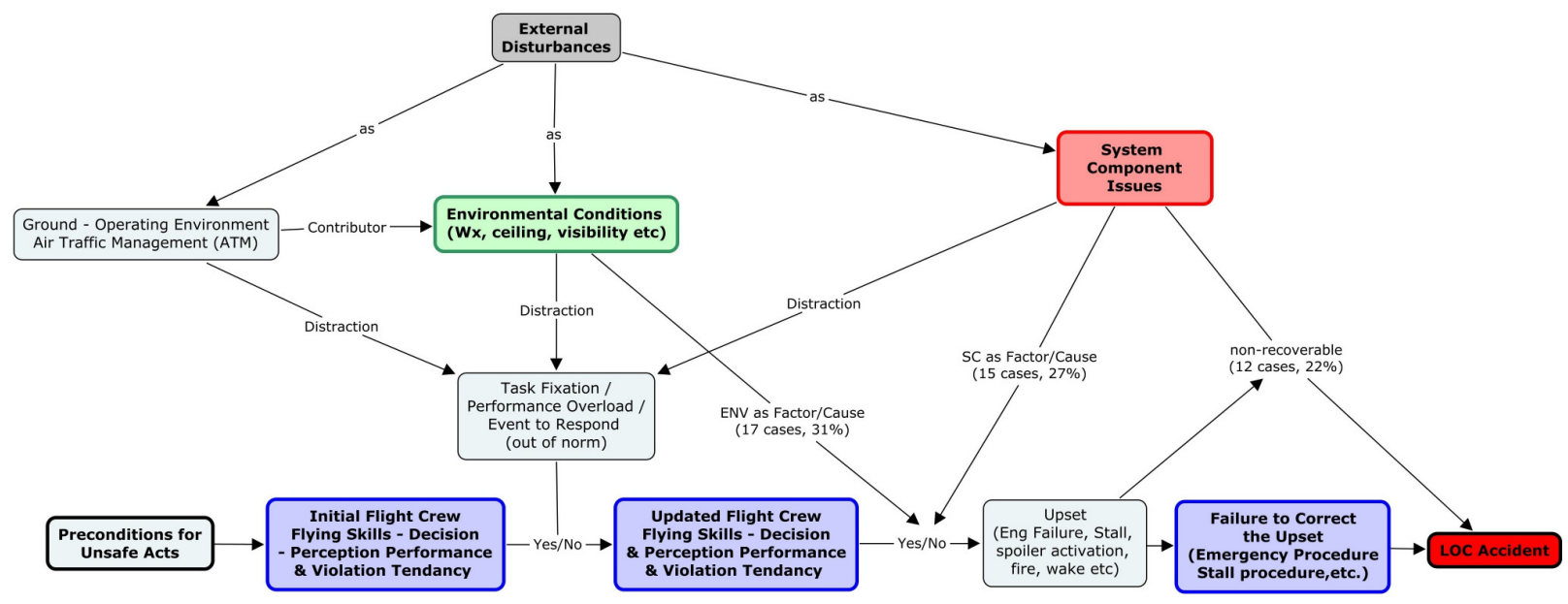

Figure 2. LOC Accident Framework (LOCAF).

The database review indicated that the aircraft system components, environmental conditions as well as ATM operations can have a negative impact on the flight crew performance. For instance, out-of-routine aircraft system indications, changing environmental conditions, or deficient ATM guidance can distract the flight crew, especially during the critical phases of flight. Such distractions, in turn, can lead to performance overload, confusion, panic, task fixation and/or saturation, captured in the Task Fixation box. However, even without the presence of such external disturbances, the flight crew can experience spatial disorientation or suffer from fatigue or medical illness. The Preconditions for Unsafe Acts box depicts initial flight crew deficiencies (a separate framework will be given in Section A, providing a more detailed review).

Once the initial flight crew performance is updated via the presence of external disturbances, the information is incorporated into to the Upset box with two other accident contributors; the system component failures and environmental factors, are taken into consideration. Environmental Conditions depicts accidents where convective weather, wake turbulence, icing, and bird strikes are determined as factor/cause. Similarly, the System Component Issues box contain failures or malfunctions that are a primary cause/factor in the LOC event. The Upset box depicts that the aircraft is an abnormal flight condition, outside the normal flight envelope. The cause for the upset can be stemmed from the the flight crew alone (e.g. loss of speed due to deficient instrumentation scan), system component 
failure (e.g. engine, hydraulic or software failure), environmental factor, or a combination of these factors. The five accident types identified in the previous section are represented in the Upset box. Finally, the flight crew's failure to correct the upset using conventional techniques will eventually lead to a LOC accident.

\section{A. Flight Crew Performance Deficiency Encapsulation using HFACS}

Historically, the Flight Crew (FC) is the most important contributor in aviation accidents ${ }^{3}$. The predispositions of the flight crew (e.g. issues with experience/training, physical or mental conditions, etc.) was given in the Preconditions for Unsafe Acts box in Fig. 2. The underlying framework for FC predispositions is captured via a modified HFACS framework ${ }^{7}$. The hierarchical causal relationship structure within HFACS allows flight crew errors to be traced all the way to the organizational level. The HFACS architecture consists of 3 levels; Operator Organizational Influences level, Operator Unsafe Supervision level, and finally the deficiency at the Flight Crew level.

In order to construct the FC performance deficiency framework, 39 flight crew related accidents (out of the original 54 cases) were analyzed and classified based on the causal factor definitions given in the HFACS taxonomy. Figure 3 provides the original HFACS causal factors and the occurrence numbers and frequencies of each factor. Despite the small sample size, the correlation between causal factor pairs is used to determine the link connecting the causal factors to each other ${ }^{\dagger \dagger}$.

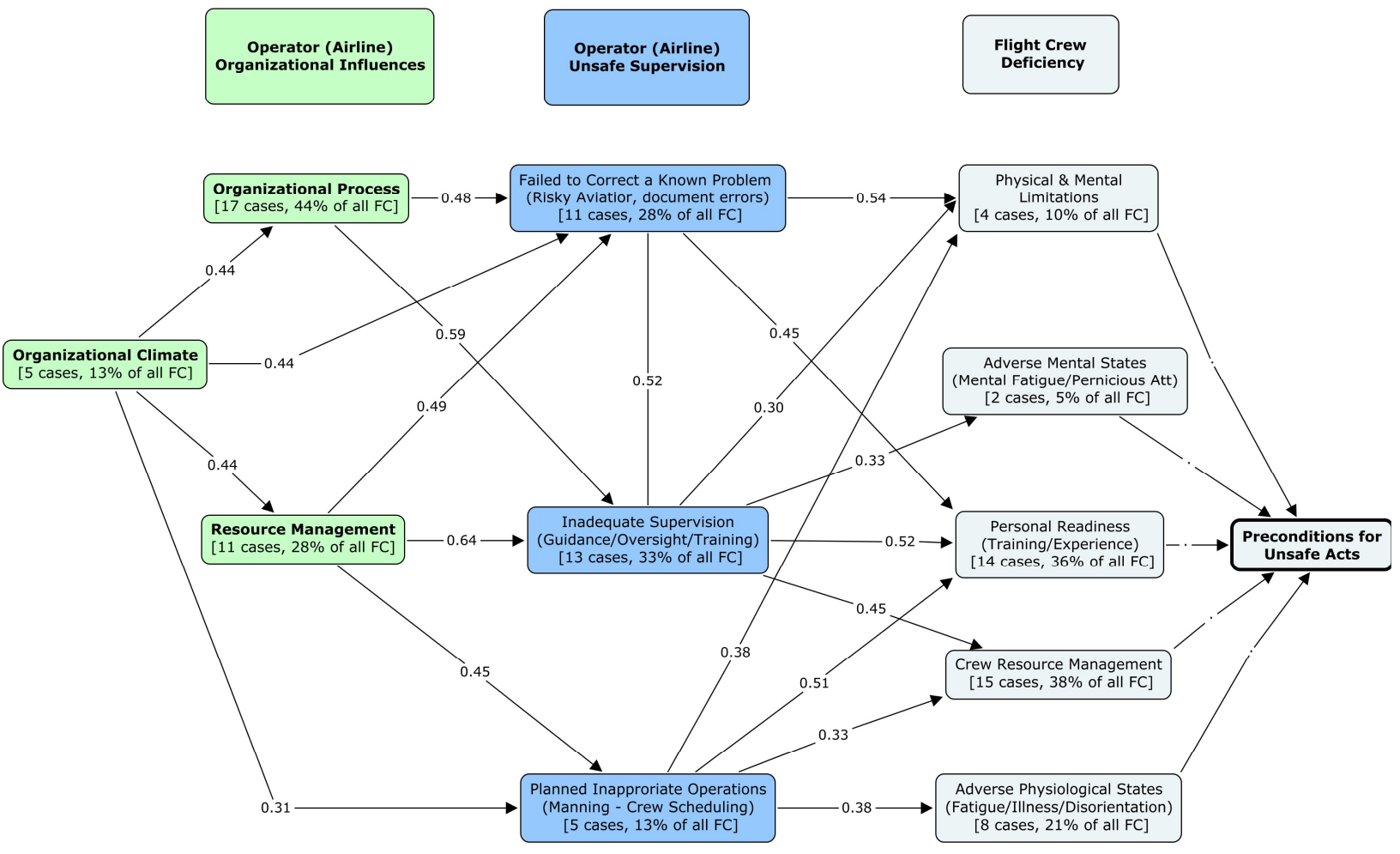

Figure 3. Flight Crew Performance Deficiency Framework based on HFACS

The organizational influences are divided in three groups; Organizational Climate, Organizational Processes, and Resource Management. The organizational climate refers to the working atmosphere within an organization as well as values and beliefs. It includes the organizational structure (chain-of-command, delegation of authority, communication practices), hiring-firing and promotion policies, and culture (norms and rules, etc.). It is argued that the individual errors within the organization can be closely related to the organizational climate 5 . Organizational climate broadly affects the organizational processes (such as the operational tempo, time pressure, production quotas, standards and procedures, documentation, risk management, safety programs oversight, etc.). Similarly,

\# The total number of flight crew accidents (39 cases) constitutes the sample size for the correlation analysis. Correlation Analysis within Excel's Data Analysis ToolPak Add-in was performed for all the accidents and results were displayed on the links in Fig. 3. 
resource acquisition management (staffing/manning, training, cost cutting, funding issues, and equipment and facility issues) is closely related to the safety understanding and safety policy which is a part of the organizational climate $^{7}$.

The organizational structure influences the supervision performance at the mid-level management. Although HFACS indicates four components within this level, the investigation did not identify supervision violations within the database. The remaining three components; Failed to Correct a Known Problem, Inadequate Supervision, and Planned Inappropriate Operations constitute the underlying causes that yield to preconditions for unsafe acts. Issues encountered with lack of oversight, training, guidance or performance tracking are associated with inadequate supervision. Inappropriate operations include cases where the crew was not allowed to rest adequately due to improper scheduling or improper manning took place. Errors in documentation, identification of risky aviators, or reporting unsafe practices are covered in Failed to Correct a Known Problem node. The influences of unsafe supervision are observed within the cases that make up the preconditions of unsafe acts. These conditions are the cases where the members of the flight crew were not good candidates (due to physical \& mental limitations) or possessed adverse mental states or pernicious attributes (over or under confidence in abilities and skills). Similarly, issues encountered with lack of training/experience as well as physiological states such as fatigue, medical illness, spatial disorientation, and crew resource management can be attributed to the combination of unsafe supervision deficiencies ${ }^{7}$, as shown in Fig. 3.

\section{B. System Component Issues}

The review of the SC related accidents (27 cases) revealed that faulty design was the primary cause of eight accidents (30\%) whereas deficiencies within the maintenance processes caused ten accidents (37\%). The remainder nine accident cases (33\%) include failures associated with avionics, instrumentation, mechanical, and other systems as well as undetermined failures that are not identified as either maintenance or design related (Fig. 4).Within the maintenance related accidents, four cases were associated with powerplant (control linkages, propellers, engine rotors, etc.), two structures (airframe, wings, stabilizers, etc.), and four flight controls (flaps, ailerons, spoilers, elevators, etc.). Maintenance related accidents were often related to the maintainer's failure to notice fatigues, cracks, corrosion pits, etc. ( 3 cases or $11 \%$ of all SC) or due to ineffective

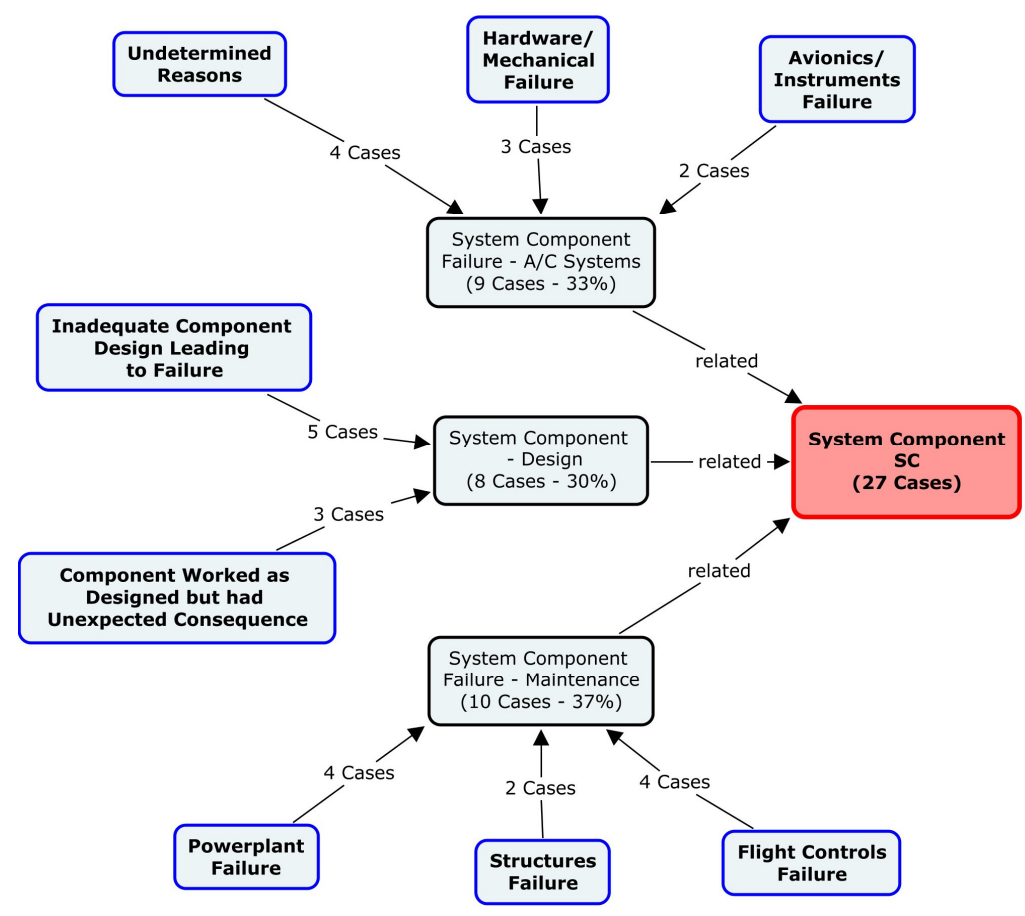

Figure 4. System Component Related Accidents maintenance (such as installing an incorrect part, misadjustment of components, incorrect rigging of flight control surfaces, etc.) (7 cases or $26 \%$ of all SC).

Similarly to the maintenance related system component failures, design related accidents were also divided in subcategories based on the presence of an actual component failure. In three cases (11\% of all SC), the system component in discussion worked as planned, but the operation caused a hazardous condition for the aircraft. An example for this category can be an inappropriate software logic design in the windshear warning system that desensitized during flap operations and failed to alert the flight crew. The other type of design failure is the actual failure of the component due to its design ( 5 cases, $18 \%$ of all $\mathrm{SC})$.

The final section of $\mathrm{SC}$ related accidents is the SCF-X, including failures/malfunctions that couldn't be 
associated with either a design or maintenance related root-cause. This subcategory is divided between hardware/ mechanical failures, avionics/ instrumentation failures, and undetermined reasons. In many cases, accident investigations does not provide information regarding the underlying cause of the failure.

\section{Environmental Conditions}

Environmental disturbances including icing, thunderstorms, turbulence and wake turbulence were either a cause or a factor in 17 accidents (31\%) of all LOC accidents (Fig. 5). It is important to note that within the database, all weather related accidents had also a flight crew error as a co-factor. The probable reason for the lack of any unrecoverable weather related accidents can be due to the omission of aircraft with MTOW less than 12,500 lbs from the database. Out of all weather related accidents, $76 \%$ include icing related problems, which occur during takeoff or in-flight. Loss of control during takeoff due to icing is caused by the presence of ice on the airframe and wings before takeoff. During the takeoff roll, the presence of ice decreases lift due to disrupted airflow around the airfoil, causing the aircraft to yaw sharply or fail to gain speed following the rotation (4 cases or 23\%).

Loss of control due to in-flight icing usually occurs on the leading-edge of the airfoil and empennage, increasing drag and decreasing lift. Degraded aircraft performance and speed eventually yield to a stall (9 cases or 53\%). Accidents related to adverse weather/wind, turbulence, and windshear present in three cases (18\%). Finally, one accident was caused by wake turbulence encounter, where a B757 aircraft was followed by a smaller IAI Westwind aircraft.

\section{Literature Review on Safety Methods and Tools}

The generalized accident framework within LOCAF was used as a platform to model LOC accidents. In order to select the modeling approach, a literature review of current safety methods and tools was conducted. A safety methods database maintained by National Aerospace Laboratory Air Transport Safety Institute ${ }^{8}$ (NLR-ATSI) in the Netherlands contains over 700 methods. These methods ${ }^{9}$ and software tools are developed for various domains of application. Review of this database revealed that more than 100 methods and tools are associated with aviation applications, to discover and prevent primary accident causes and prevent them in the future. However, rather than an exhaustive review of all the identified methods, only certain popular methods and their key components were investigated and compared.

Pertaining to aviation safety risk assessment, the prominent methods include the Aviation Safety Risk Model $(\mathrm{ASRM})^{10}$, the Causal model for Air Transport Safety $(\mathrm{CATS})^{11}$, and general Probabilistic Risk Assessment (PRA based on mainly event tree and fault tree analysis). The development of ASRM was supported by the FAA and the NASA's Aviation Safety Program (AvSP), whereas CATS was supported by the Netherlands Ministry of Transport. CATS employs a combination of three modeling techniques, Event Sequence Diagrams (ESDs), Fault Trees (FTs) and Bayesian Belief Networks (BBN), whereas ASRM uses only BBNs. The ESDs and the FTs in CATS, though developed separately, are converted into BBNs, and the final risk model is mathematically represented as a single integrated BBN. CATS employed UNINET software to program BBNs, while Hugin was the software choice for ASRM.

For this study, the key objective is to develop a system-level integrated LOC model to examine the integration of AvSP portfolio elements to address the future risks of LOC. The LOC accidents rarely result from a single linear causal sequence due to interactions between aircraft system, humans and external environment variables. Therefore, a BBN is a more intuitive and appropriate method for this large complex integrated LOC model than traditional FT and ET methods that do not easily capture the multiple non-linear dependencies. In the BBN framework, probabilistic and causal relationships among variables are flexibly represented and executed as graphs, and can thus be visualized and easily modified. This facilitates model building, and rapid interactive manipulations of the model to explore the causal features, which are particularly helpful when interacting with Subject Matter Experts (SMEs) in the probability elicitation process. 
There are many different BBN software packages ${ }^{\ddagger}$ available from both commercial vendors and the publicdomain, such as BNet, Hugin, Netica, WinBUGS. Hugin software has many advantages over competing tools for the purpose of this modeling endeavor ${ }^{12}$. Hugin is favored mainly due to three reasons: its critical capability of employing Influence Diagrams (ID), the Object-Oriented Bayesian Network (OOBN) feature, and the availability of an Application Program Interface (API). ID is an effective means to evaluate the impact of new safety technologies on the reduction of aviation safety system risk, providing decision makers useful information for technology portfolio management. The OOBN feature allows construction of a modular and hierarchical BBN for more efficient modeling, and succinct presentation of a large and complex BBN. The Hugin API is available for most major programming languages, such as $\mathrm{C}, \mathrm{C}++$, Java, etc. so that additional modules can be developed to enhance the core BBN model component.

\section{BBN-based Generic LOC Model with Human Error Emphasis}

A previous NASA-guided study ${ }^{10}$ with a similar goal of evaluating the effectiveness of NASA safety technology products employed case studies to represent various types of accidents (e.g. LOC, controlled flight into terrain, runway incursion, engine failure, etc.). However, the prominence of human factors, especially the human error interactions in all types of LOC accidents, exceeds the ability of a case study approach to be used here.

The Hugin BBN-based generic LOC model allows the authors to capture conditional probabilities (elicited from SMEs) to compute the likelihood of LOC. More importantly, the generic LOC model provides a platform to evaluate the effectiveness of currently developed NASA safety technology products within the VSST, AEST, and SSAT projects while allowing enough flexibility to the authors to modify the generic human error model to address future concerns without being restricted to any specific case study. For that reason, the previously defined LOCAF framework (Fig. 2) was converted into a Bayesian based Object Oriented model using Hugin Software. The use of OOBN simplifies construction and maintenance of this large and complex LOCAF model.

Fig. 6 provides the causal factors of the accident, represented via nodes and connected by links, designating dependencies at the top-level of the LOCAF model. The ellipse nodes are called discrete chance nodes and the each node's dependency on its parent nodes are given with a Conditional Probability Table (CPT). CPTs include all the possible combinations of nodes' parents and the respective probability values used in Bayesian calculations.

The rectangle nodes with rounded corners in Fig. 6. represent instance nodes, indicating they are encapsulated. The encapsulated nodes include Flight Crew Conditions, Environmental, and System Component Failures and each of these nodes contains their own separate BBN (also called subnets) which are fed to this level. The application of object-oriented encapsulation allows each subnet to be enhanced or replaced easily, as well as the addition of new

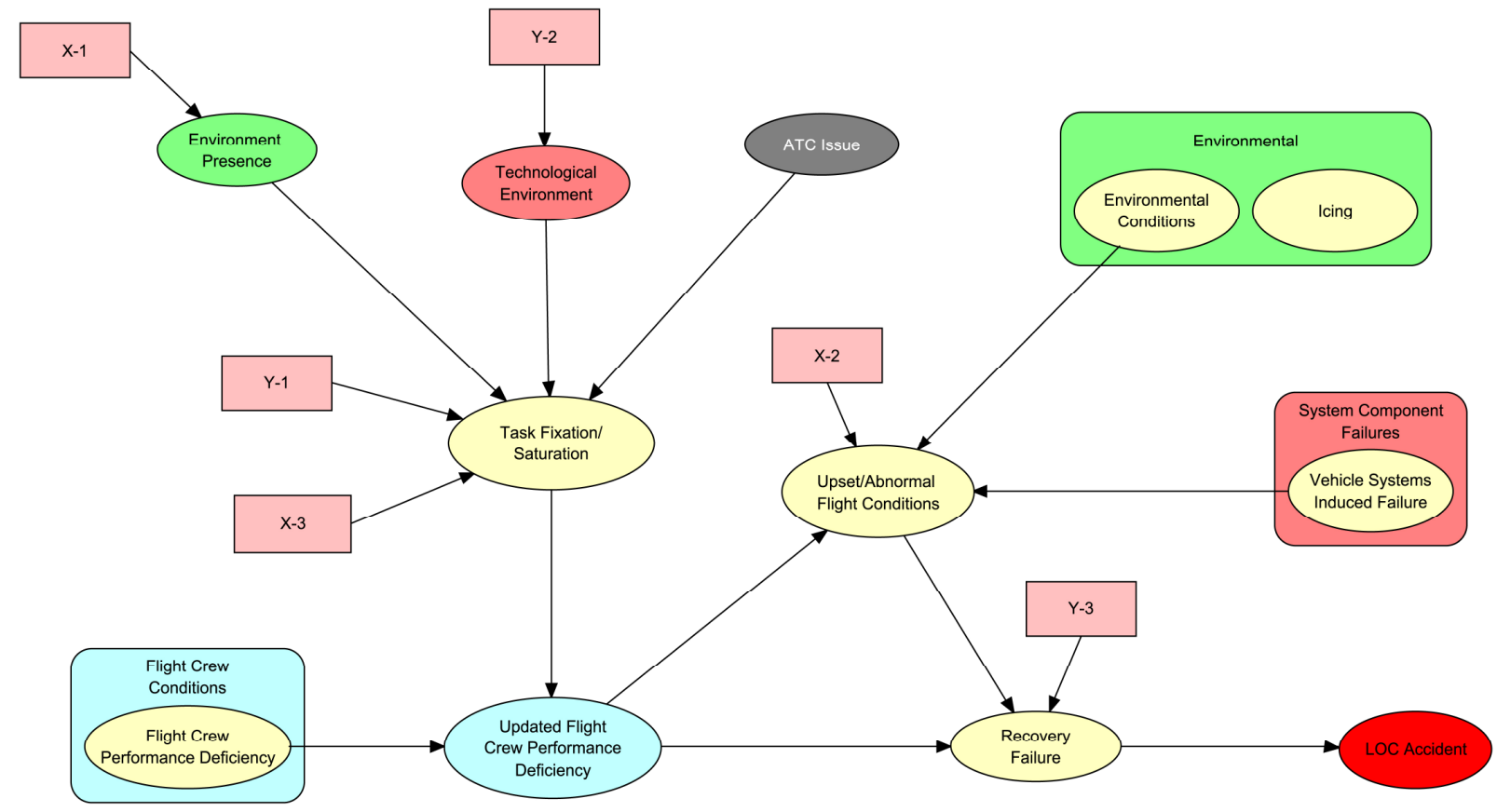

Figure 6. Top-level LOCAF Model Representation using Hugin

American Institute of Aeronautics and Astronautics 
sub-models, without disturbing the rest of network in the future. Within the scope of this paper, only the flight crew conditions and system component failures associated with faulty maintenance (SCF-M) will be provided in detail within Sections V-A and V-B. The model shown in Fig. 6 coupled with the encapsulated nodes constitute the baseline LOC model. The details and the preliminary results of the baseline model will be discussed in Section V-C. The rectangle nodes are called decision nodes and they are used to represent various NASA products and technologies (X-1, Y-2, X-3, etc.). It should be noted that the product label and the placement of the decision nodes in Fig. 6 are notional. Nevertheless, the process of product insertion and evaluation will be covered in Section V-D.

The encapsulated Flight Crew Conditions node provides the calculated probability of deficiency in flight crew's initial performance. The presence of deficiency indicates the probability of flight crew errors (skill, decision, and perception) and violations in an accident. This node depicts the flight crew's preexisting conditions before the flight and is covered in more detail in Flight Crew Performance Deficiency section.

The Task Fixation/Saturation is an aggregating node, combining the possible effects of external influences on the flight crew. It covers the presence of environmental factors (e.g. presence of low-ceiling, wind gusts, or icing conditions), discrepancies with the technological environment (interactions with the a/c, autopilot/automation, cockpit/checklist design, etc.), and the ATC related issues (increased workload caused by inadequate ATC procedures/communication or due to high traffic density). The resultant effect is calculated within the task fixation/saturation node which has three states; fixation, saturation, no effect. Environment, technological environment and ATC issue nodes are two-state nodes where the effects of each node on an accident are obtained from SMEs.

The Updated Flight Crew Performance Deficiency node provides the flight crew performance deficiencies during the flight by taking various effects into consideration. The link from the Task Fixation/Saturation node is fed into the Updated Flight Crew Performance Deficiency in order to calculate the ongoing flight crew performance deficiency which may be impaired from the effects of fixation/saturation. In other words, any presence of task saturation/fixation effect is aggregated when calculating the updated flight crew performance deficiency. It was often observed that the presence of external effects such as low visibility or increased traffic workload were not directly related to accidents but may have contributed to flight crew's performance degradation.

The Upset/Abnormal Flight Conditions node is another aggregating node where inputs from System Component Failures (SCF), Environmental factors, and FC performance subnets are evaluated to provide the probability of an upset/abnormal flight condition occurrence. An upset condition includes engine failure, fire, stall, wake, uncontrolled altitude deviation, unresponsive aircraft, etc. where one or more parent nodes can be a factor. For example, a deficiency in flight crew's skill can result in a loss of aircraft energy (low-speed) which can cause a stall. Similarly, a SCF within the power-plant or aircraft controls can render the aircraft uncontrollable, which in turn yield to an abnormal flight condition. The encapsulated environment node includes the presence of wake turbulence, convective activity, icing and wildlife/bird strike. Similarly SCF provides failure probabilities of a generic aircraft that contributes in an accident scenario.

The Recovery Failure node (successful/unsuccessful recovery) refers to the ability of the flight crew to recovery from an abnormal flight condition. Failure to recovery inherently leads to a LOC accident, where a successful recovery indicates the absence of LOC. Inherently, deficiencies in the flight crew's performance are directly related to the recovery failure (i.e. following an emergency procedure, flying skills, training, etc.).

\section{A. Flight Crew Performance Deficiency Subnet}

The flight crew performance deficiency framework which was based on a modified version of the HFACS ${ }^{5,7}$ architecture was given in Fig 3. The contents of the initial framework was used as a baseline for the development of flight crew performance deficiency subnet as shown in Fig. 7. This subnet was then evaluated and modified by the SME panel ${ }^{13}$. This subnet provides the initial flight crew deficiency probability to the top-level LOCAF given in Fig. 6 using Hugin's output node function.

The Flight Crew Performance Deficiency subnet is divided into three organizational hierarchy levels (from leftto-right); airline organizational influences (Organizational Climate, Organizational Process, Resource Management), mid-level management/supervision (Failed to Correct a Known Problem, Training, Inadequate Supervision, Inappropriate Operations), and finally the flight crew's conditions (Personal Readiness and CRM). 


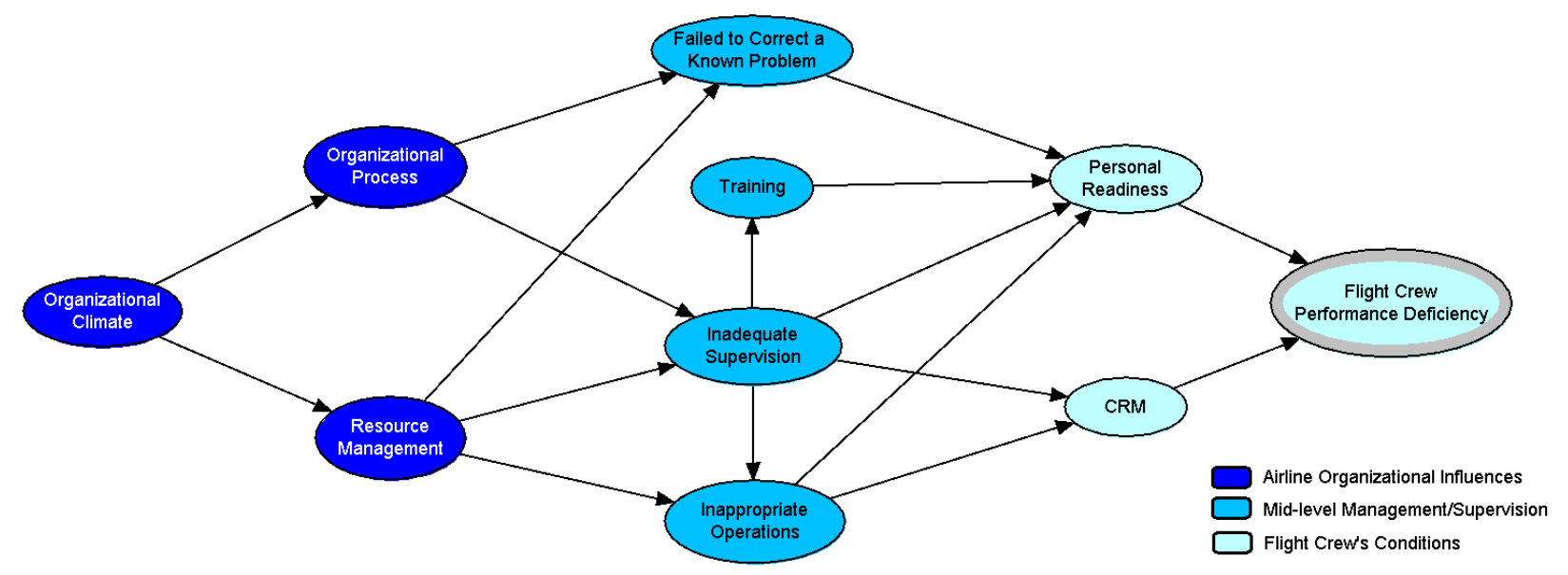

Figure 7. Flight Crew Performance Deficiency Subnet

\section{Airline Organizational Influences}

The Organizational Climate node designates the working atmosphere within the airline company. It refers to the efficiency with which the processes related to organizational communication, chain-of-command, and delegation of authority are performed. Also the organization's safety culture (unspoken rules, norms and values, attitude towards safety) and policies (official guidelines such as promotion, retention, sick leave, etc.) are covered under this node. This node contains two-states; probability of Organizational Climate Issues, $x$, and the absence of organizational climate issues, $1-x$. The values for the two states are obtained from the SMEs.

The Organizational climate sets the tone for both organizational processes and resource management in the airline company. Organizational Processes involve corporate decisions and rules that govern everyday activities within an airline company including operations, oversight, and procedures. (e.g., organizational tempo, incentives, time pressures, and schedules, safety/risk program establishment performance standards, documentations, and instructions. The two-state nature of this node points out deficiencies on airline's organizational processes (present or absent).

The Resource Management node refers to the corporate-level decision making regarding the allocation and maintenance of organizational assets such as human resources (e.g. personnel selection, staffing/manning, training), monetary assets, facilities, and equipment. Examples of deficiencies associated with underperforming resource management can be given as lack of funding, excessive cost cutting, opting for low-cost but sub-optimal alternatives, inexperienced flight/maintenance abundance, etc.

\section{Supervision Level Influences}

Based on the review of the accidents, it was observed that deficiencies at high-level organizational management propagated to the supervision level. At the supervision level, the Failed to Correct a Known Problem node refers to the instances when known deficiencies among individuals, equipment, operations, procedures or other safety areas are left untreated. Supervision's failure to identify and correct the risky behaviors, safety hazards and failure to initiate and follow up corrective action is categorized under this node.

The Training node covers deficiencies associated with a supervisors' failure to provide adequate training due to cost cutting, scheduling conflicts, or other reasons. Also included in this node are instructional errors or omissions during training as well as inappropriately planned simulator and flight training causing the pilot not to receive appropriate knowledge and skill set necessary to safely fly the aircraft.

The Inadequate Supervision node reflects supervisory deficiencies that take place within organizations. Supervisors' duties include providing guidance, leadership, oversight, and incentives in order to ensure the efficient and safe working environment. Examples of inadequate supervision can be cited as failure to provide an adequate rest period, lack of accountability, loss of supervisory situational awareness, and failure to track qualification and performance of employees.

The Inappropriate Operations node considers deficiencies associated with the operational tempo and/or scheduling of the aircrew. Inappropriately planned operations put the flight crew (and the passengers) at an unacceptable risk since crew rest is jeopardized and ultimately performance is adversely affected. This node also includes poor crew pairing (seniority issues), excessive tasking/workload, failure to provide adequate brief time, etc. 


\section{Flight Crew's Conditions}

Although historical data provided statistical correlation on the presence of various flight crews' preconditions for unsafe acts, the SMEs re-organized this section of HFACS to contain two parts; Personal Readiness (covering all the physical and mental preconditions) and $C R M$ related deficiencies.

The Personal Readiness node includes all long and short term physical and mental readiness of the flight crew as well as the experience and training deficiencies that may be present. Examples of mental limitations and states include complacency, distraction, get-home-it-is, misplaced motivation, mental tiredness, distraction, confusion, depression, alcoholism, over/lack of confidence. Similarly, examples of physical states and limitations include physical fatigue (lack of sleep or flying schedule), medical illness, excessive physical training, impaired physiological state, physiological incapacitation, self medication, violation of crew rest requirement or bottle-tothrottle requirement. Finally, individuals that are not compatible with aviation (physically or mentally unable to process, react of fit to fly due to insufficient reaction time, visual limitation) are also covered in this node.

The second precondition for unsafe act besides the Personal Readiness is Crew Resource Management (CRM) deficiencies. The $C R M$ node includes communication skills and coordination that take place within the flight crew as well as between other entities (i.e. other aircraft, ATC, maintenance facility and other support personnel) before, during, and after the flight. Regardless of the experience of the pilots, poor CRM and coordination can lead to confusion and poor decision making in emergency or trouble shooting situations.

The resultant deficiencies within the pilot's personal readiness and CRM issues determine the value of the Flight Crew Performance Deficiency node. This node is the output node of the Flight Crew Performance Deficiency subnet and it takes the Bayesian probability values from the parent nodes to calculate the preconditions of the flight crew. A deficiency in the flight crew performance is defined as flight crew's tendency to make errors and commit violations. The flight crew errors can be associated pilot's skills, decision making abilities as well perceptions (e.g. stick-and-rudder capabilities, deficient instrument scan, takeoff/landing decision, improper flight planning, misjudging distance, descent, altitude, spatial disorientation, vertigo, etc.).

Unlike errors, violations are performed willingly by bending the rules habitually or in isolation. The Flight Crew Performance Deficiencies node captures all the error and violation types covered above and indicates the flight crew's tendancy to perform unsafe acts. As previously mentioned, this provides the initial conditions of the pilots at the top-level LOCAF model and it is "updated" with the Task Fixation/Saturation node.

\section{B. Maintenance Related System Component Failure (SCF-M) Subnet}

Within the scope of this paper, accidents caused by faulty maintenance are taken into consideration due to the human factors involved. The SCF-M subnet is a simplified and modified version of the HFACS-Maintenance Extension (HFACS-ME) ${ }^{6}$. Similar to the HFACS framework, the maintenance extension considers latent conditions such as supervisory deficiencies, maintainer's preconditions (such as readiness, fatigue/illness, etc.) as well as the working environment when analyzing the maintenance related system component failures. Like the flight crew preconditions, any deficiency present in the maintainer's conditions can cascade as an error in one of the four generic tasks (adjustment, installation, service of aircraft, and inspection). This subnet covers the repair stations as well as the corporate airlines maintenance departments (Fig. 8). 


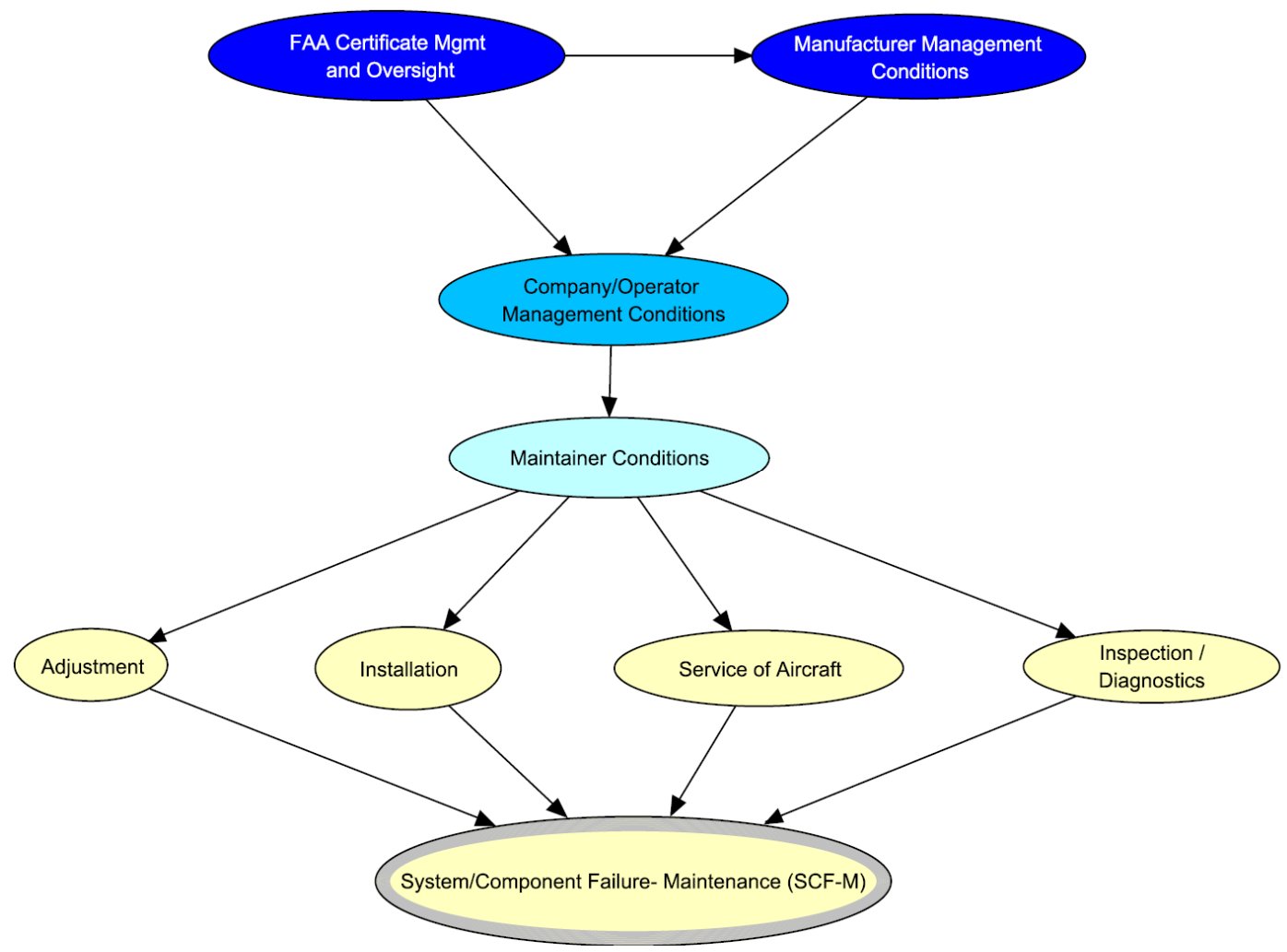

Figure 8. System Component Failure related to Maintenance (SCF-M) Subnet

In order to capture system failures caused by faulty maintenance, this subnet takes into account deficiencies within the original equipment manufacturers (OEM), FAA, and airline operators and/or repair stations. The following paragraphs provide an overview of the nodes involved in the hierarchy.

The FAA Certificate Management and Oversight node covers the efficiency with which FAA oversight is applied when enforcing rules and regulations such as the Parts 145 (Repair Stations), 21 (Certification Procedures for products and parts), 25 (Airworthiness Standards - transport category) as well as other maintenance related processes. The FAA's oversight mandates the compliance of maintenance departments, repair stations as well as OEMs to these regulations. Like the rest of the nodes in this subnet, the two-states designates potential deficiencies in the nodes' function (Yes/No).

The Manufacturer Management Conditions node refers to the management conditions at the aircraft manufacturers, vendors and part suppliers. This node captures the deficiencies within the manufacturer management conditions which may lead to inadequate information flow to the end users (airline operators, repair stations) regarding the maintenance practices (e.g. late issued service letters/advisory circular/service bulletins, unclear maintenance instructions, etc.) Consequently, poorly communicated maintenance requirements can result in inappropriate maintenance procedures to be performed.

The Company/Operator Management Conditions node captures the entire management deficiencies that can be encountered in maintenance departments and repair stations. Like the manufacturing companies, maintenance departments are also mandated by FAA regulations. Inappropriate FAA oversight (conducted via Repair Station Certificate) can cause deficiencies within the repair facility to go unnoticed. Similarly, any problems in guidance and communication of the OEM can play a significant role in maintenance related system component failures (e.g. relaying information regarding changes to the maintenance processes, materials, specifications, etc). For that reason, the Company/Operator Management Conditions are affected by both FAA Certificate Management and Oversight and by deficiencies stemmed from Manufacturer Management Conditions. Issues within the Company/Operator Management Conditions can be given as inadequate documentation of requirements/ processes, resources (e.g. lack of job specific tools and equipment, maintenance crew qualifications and training) as well as inappropriate implementation of the regulations (e.g. omitting steps in procedures) and supervisory issues.

The Maintainer Conditions node covers all the physiological and mental condition (readiness, training, experience) of the maintainer (e.g., mechanic, electrician) as well as the working conditions (environment, 
equipment, and workspace). The Company/Operator Management conditions directly affect the aspects of the maintainers' conditions via supplying adequate tools, guidance, and documentation as well as providing sufficient time and training for the job. Consequently, deficiencies encountered at the airline's maintenance department or repair facility can be related to the technicians less-than-adequate performance. Issues in the technicians' performance include potential errors (due to lack of attention, knowledge, skill or decision making) and violations (routine or exceptional). This node is synonymous to the Personal Readiness node found in the flight crew performance subnet.

The Adjustment/Installation/Service of Aircraft/Inspection (Diagnostics) nodes include the common maintenance practices routinely performed on the aircraft. Their goal is to bring the specifications of the part/subsystem to the OEM requirements. The aircraft often goes through a non-destructive inspection where the deficiencies are detected and the subsystems/parts are installed and/or adjusted. Also, the aircraft undergoes several scheduled (line checks) and unscheduled (pilot initiated maintenance write-ups) checks, which are captured within the service of aircraft node. The deficiencies found at the maintainer level can have adverse effects on how these four maintenance processes are performed and the varying probability values are obtained via SME sessions.

\section{Baseline Model Results}

As previously mentioned, the SME sessions were conducted during the construction of the LOCAF model ${ }^{13}$, reviewing the nodes and the linkeages, as well as collecting the probability data for the CPTs. Subsequent to the conversion of LOCAF to the BBN and data collection via SME sessions, the resulting model without product insertion provides the representative baseline for LOC accidents. The preliminary results of the model showed that individual subnets, as well as the top-level LOC model was within approximately $3 \%$ of the historical data with which the model was constructed. Following the calibration/adjustment of the raw data supplied by the experts, the LOCAF model was compared with the results obtained from two datasets ${ }^{\S \S}$. The overall LOC probability was found to be $15.9 \%$ (versus historical $13.8 \%$ ), and $10 \%$ (versus historical $12.8 \%$ ) in the two datasets . The object-oriented structure of the model allows authors to test various scenarios where individual subsystems coupled with various environmental factors impact on the LOC occurrence. This detailed analysis of the collected data will be further analyzed in the later stages of the research.

\section{The Assessment of AvSP Products on LOC}

In addition to computing the likelihood of LOC, the LOCAF model was developed to evaluate the overall effectiveness of over forty NASA developed technologies and methodologies (referred to as products) within the AvSP. The generic product insertion is a three-step process. First, similarly to the model construction and data collection phases, the NASA products was reviewed by the same set of SMEs ${ }^{* * *}$ using product descriptions and other documents provided by AvSP Projects. Once the experts obtained a common knowledge over the NASA products developed under VSST, AEST, and SSAT Projects, the next phase involved the placement of these products into the LOCAF model. The product insertion involves linking the decision node(s) (rectangular nodes, as shown in Fig. 6) to causal factors (chance nodes) within the model. The decision nodes have two states; designating the presence or absence of the product. The third step of the product insertion process involved acquiring the new deficiency probabilities of the nodes considering the presence of the product. The products are assumed to be $100 \%$ effective, as intended by their designers. The effect of the product on the causal factor is captured within the respective node's CPT. The future phases of this study will include the assessment of various combinations of AvSP portfolio elements to determine their effectiveness by calculating the relative change on LOC.

\section{Conclusions}

Based on historical data, LOC accidents play a significant role in fatal commercial aviation accidents. This paper encapsulates the comprehensive process of a generic LOC model building effort which enabled the evaluation of the impacts of various NASA AvSP products. The process of model building was initiated with the extensive review of a limited, but significant dataset of accidents from 1987 to 2009. The inferences and knowledge acquired from the historical data review were used to construct a generic LOC accident framework, which was supported by HFACS and HFACS-ME frameworks aiming to capture the human errors encountered with flight crew and faulty

\footnotetext{
$\S$ The databases used for comparison are the 54 accident cases employed to construct the model as well the dataset covered in Ref. 3 (Part 121/135, 1988-2004).

${ }^{* * * *}$ The three experts who participated in the model development and data collection session were also invited to insert and evaluate NASA developed AvSP products for consistency purposes.
} 
maintenance, respectively. The analysis of the historical data showed that the deficiencies at the airlines' organizational levels are often the underlying cause of flight and maintenance crew related accidents. Consequently, the authors developed a high-level airline organizational hierarchy to trace and identify the deficiency propagation.

The causal accident factors and their interdependencies were constructed using historical data as well as aforementioned human factors framework suites. The resultant accident framework was then converted into a OOBN using the Hugin Software, which enabled the authors to develop different network fragments of the model (human error, system component failure, etc.) separately, then merging to form a generic LOC model. The application of OOBN on the LOCAF model allows authors to export entire subnets (e.g. flight crew performance subnet) to other OOBN accident models for a broader accident/incident modeling portfolio. The preliminary results indicate that the SME-supported LOCAF model with human factors emphasis is adequate for representing LOC accidents, as well as providing a platform for gauging various NASA technologies' effects on decreasing future LOC occurrences.

\section{Acknowledgment}

The authors would like to thank the entire Aviation Safety Analysis Team, specifically, Dr. Sharon M. Jones, Mary Reveley, Lawrence Green (NASA), Joni Evans (AMA, Inc.), and Dr. James Luxhøj (LCR) for their contributions to the development of LOCAF model. We are also very grateful to the subject matter experts for their participation in the knowledge elicitation sessions and insightful discussions on the model.

\section{References}

\footnotetext{
${ }^{1}$ Boeing Commercial Airplanes, "Statistical Summary of Commercial Jet Airplane Accidents, Worldwide Operations, 19592010," June 2011. URL: http://www.boeing.com/news/techissues/pdf/statsum.pdf. [Accessed December 2011].

${ }^{2}$ Jones, S. M., Reveley, M. S., Withrow, C., Evans, J. K., Barr, L. C., and Leone, K., "System Analysis of NASA Aviation Safety Program - Final Report," (Draft), Hampton, VA, June, 2010.

${ }^{3}$ Evans, J. K., "An Application of CICTT Accident Categories to Aviation Accidents in 1988-2004," NASA CR-2007$214888,2007$.

${ }^{4}$ CAST/ICAO Common Taxonomy Team, "Aviation Occurence Categories Definitions and Usage Notes," Version 4.1.5, April 2011.

${ }^{5}$ Shappell, S. T., and Weigmann, D. A., "The Human Factors Analysis and Classification System," U.S. Department of Transportation Office of Aviation Medicine, DOT/FAA/AM-00/7, Washington, DC, February, 2000.

${ }^{6}$ Schmidt, J. K., Lawson, D., and Figlock, R., "Human Factors Analysis \& Classification System - Maintenance Extension (HFACS-ME) Review of Select NTSB Maintenance Mishaps: An Update," $2003 . \quad$ URL: https://www2.hf.faa.gov/dtsearch/dtSearch.html.

${ }^{7}$ Wiegmann, D. A. and Shappell, S. A., A Human Error Approach to Aviation Accident Analysis: The Human Factors Analysis and Classification System. Surrey, UK: Ashgate, 2003

${ }^{8}$ NLR, "Safety Methods Database," December 2010. URL: http://www.nlr.nl/documents/flyers/SATdb.pdf.

${ }^{9}$ Everdij, M. H. C., Blom, H. A. P., and Kirwan, B., "Development of A Structured Database of Safety Methods," Proceedings of the 8th International Conference on Probabalistic Safety Assessment \& Management (PSAM8), New Orleans, LA, May 14-18, 2006.

${ }^{10}$ Luxhoj, J. T., Jalil, M., and Jones, S. M., "A Risk-Based Decision Support Tool for Evaluating Aviation Technology Integration in the National Airspace System," Proceedings of the AIAA's 3rd Annual Aviation Technology, Integration, and Operations (ATIO) Technical Forum, Denver, CO, November 17-19, 2003.

${ }^{11}$ NLR Air Transport Safety Institute, "Causal Model for Air Transport Safety, Final Report," Amsterdam, NL, 2009.

${ }^{12}$ Shih, A. T., Ancel, E., Jones, S. M., "Object-Oriented Bayesian Networks (OOBN) for Aviation Accident Modeling and Technology Portfolio Impact Assessment" Proceedings of the American Society for Engineering Management (ASEM) 33 ${ }^{\text {rd }}$ International Annual Conference, Virginia Beach, VA, October 17-20, 2012 (submitted for publication).

${ }^{13}$ Luxhoj, J. T., Shih, A. T., Jones, S. M., Ancel, E. and Reveley, M. S., "Safety Risk Knowledge Elicitation in Support of Aeronautical R\&D Portfolio Management: A Case Study," Proceedings of the American Society for Engineering Management

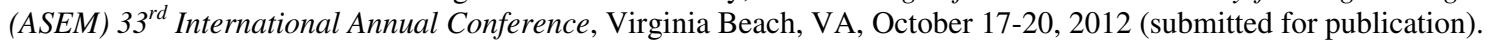

